Research Paper

\title{
Lymphangiogenesis in Classical Hodgkin Lymphoma - Preliminary Study with Clinicopathological Correlations
}

\author{
Daniel Benharroch $^{1 凶}$, Isebrand Prinsloo ${ }^{1}$, Jacob Gopas 2,3 , Irena Lazarev² \\ 1. Departments of Pathology, Soroka University Medical Center, and Faculty of Health Sciences, Ben-Gurion University of the Negev, Beer-Sheva; \\ 2. Departments of Oncology, Soroka University Medical Center, and Faculty of Health Sciences, Ben-Gurion University of the Negev, Beer-Sheva; \\ 3. Department of Immunology, Microbiology and Genetics, Faculty of Health Sciences, Ben-Gurion University of the Negev, Beer-Sheva, Israel. \\ $\triangle$ Corresponding author: Prof. Daniel Benharroch, Department of Pathology, Soroka University Medical Center, 1, Izhak Rager Boulevard, P.O.Box 151, \\ Beer-Sheva 84101, Israel. Tel. +972507579140. e-mail: danielbenharroch1@gmail.com.
}

( ) Ivyspring International Publisher. Reproduction is permitted for personal, noncommercial use, provided that the article is in whole, unmodified, and properly cited. See http://ivyspring.com/terms for terms and conditions.

Received: 2016.06.05; Accepted: 2016.08.14; Published: 2016.10.23

\begin{abstract}
A role for lymphangiogenesis in metastatic breast and prostate cancers has been suggested recently. The relevance of lymphangiogenesis in cancer as a rule, and more specifically in classical Hodgkin lymphoma, is poorly understood in comparison with that of angiogenesis. In a preliminary (pilot) study we have investigated the role of lymphatic vessels growth in 19 cases of classical Hodgkin lymphoma stained with the D2-40 (podoplanin) antibody. In each case, three lymphatic vessels hot spots were scrutinized twice. Of the 57 hot spots thus identified, we chose 15 at random for photography, microvessel counting and image analysis. We determined the mean perimeter, surface area, major axis length and complexity factor for each hot spot and correlated them with clinical and biological features of classical Hodgkin lymphoma. No correlations were found with clinical features. No associations were noted with the standard immuno-markers of classical Hodgkin lymphoma. However, significant inverse correlations were shown with $\mathrm{pRb}, \mathrm{BAX}$ and IKB- $\alpha$ expression. The mean lymphatic major axis length was inversely correlated with the complexity factor. Last, we carried out an additional clinicopathological correlation of the expression of pRb, BAX and IkB- $\alpha$ in a cohort of classical Hodgkin lymphoma patients previously published.
\end{abstract}

Key words: Lymphatic vessels; classical Hodgkin lymphoma; pRb; BAX; IкB a; complexity factor.

\section{Introduction}

Angiogenesis or proliferation of new blood vessels, is one aspect of cancer therapy which have raised controversy. It has been investigated both in primary malignant tumors, like lung, breast and colon cancers, as well as in their lymph node metastases [1, 2]. Considered as a mechanism by which increased dissemination of malignancies is facilitated, it has led to attempts to limit the neoplastic growth by inhibiting blood vessels proliferation [3]. These efforts have not been significantly rewarded to a major extent [4].

More recently, it was suggested that lymph vessels growth or lymphangiogenesis may be apt to fulfill the expectations to a higher degree, where angiogenesis had failed [5].

Classical Hodgkin lymphoma (cHL) is a primary malignant tumor of the immune system. It involves almost exclusively lymph nodes at diagnosis. Whereas angiogenesis and later lymphangiogenesis encompassing lymph nodes, have been shown predominantly in metastatic tumors, they occasionally have been described in primary cancers of lymph nodes, in malignant lymphomas [6, 7]. Rarely, angiogenesis has been investigated in Hodgkin lymphoma. These instances have been explored either with morphometric methods, used to determine the microvessel density, defined by means of aggregates of small vessels, or hot spots, correlated it with clinicopathological features [8]. They may also have been probed by evaluating the expression of VEGF and variants thereof in Hodgkin-ReedSternberg tumor cells of cHL. To date, a single paper 
has approached to a sufficient degree the definition of lymphangiogenesis in cHL [9].

We set out to clarify, in a pilot study, the characteristics of lymphangiogenesis in $\mathrm{CHL}$, using a morphometric technique, as an indication of its clinical and biological significance in this malignancy.

\section{Material and Methods}

Nineteen cases of mixed-cellularity cHL primarily involving lymph nodes were preliminarily selected from our archives, as nodular sclerosis cHL is more difficult to analyze, due to the wide fibrous bands. In order to demonstrate the lymphatic vessels $(\mathrm{LV})$ in formalin-fixed, paraffin-embedded tissue sections, 5 microns thick, we carried out immunostaining with the podoplanin, D2-40 antibody (1:100, Dako) using the avidin-biotin complex method. In each case, three LV hot spots were selected, a total of 57 hot spots. The hot spots were photographed at 200X magnification and printed in black ink on white paper. The LVs were traced onto the photographs: the LV outlines in green and the LV endothelial lining in red.

Of the 57 hot spots prepared, fifteen chosen at random, were submitted twice for morphometry. In each case, the mean LV perimeter, surface area, major axis length was evaluated and a mean complexity (shape) factor was calculated, using the microvessel counting and image analysis described by Korkolopoulou et al (8). The complexity factor was calculated as equal to $4 \Pi^{*}$ area/ perimeter ${ }^{2}$. The above LV parameters were correlated with clinical features, including age, gender, ethnicity, stage, systemic symptoms and disease outcome. They were also correlated with CD30, EBV (LMP1 and EBER), CD20, p53, MDM-2, pRB, BAX and ІкB- $\alpha$ expression and with the apoptotic index [10].

The statistical analysis used the Mann-Whitney test for non-parametric values and Spearman's analysis for non-parametric correlations.

In 10 separate sections of the hot spots, we carried out a double staining in which the LVs were stained with D2-40 and counterstained with Diaminobenzidine (DAB) and blood vessels were demonstrated with the CD34 antibody and counterstained with the AEC substrate. Double staining was based on the method described by Kyzas et al [11]. Photographs of the double staining contrast the blood vessels stained in red and LV in brown.

Last, we looked for clinicopathological correlations regarding $\mathrm{pRb}, \mathrm{BAX}$ and IKB- $\alpha$ gene products, identified in the first part, as relevant for lymphangiogenesis, in the pilot study. Associations between the gene expression were determined with clinical and biological features in a cohort of previously analyzed cHL patients [12].

\section{Results}

\section{Lymphangiogenesis in classical Hodgkin lymphoma, the pilot}

Lymphatic vessels stained brown with D2-40 (podoplanin) (Figure 1a). A double staining highlighted blood vessels in pink-red (CD34 with AEC substrate) and lymphatic vessels in brown (D2-40 with DAB substrate - Figure 1b). Scarce HRS cells did not demonstrate a specific configuration relationship with lymphatic vessels (Figure 1c).

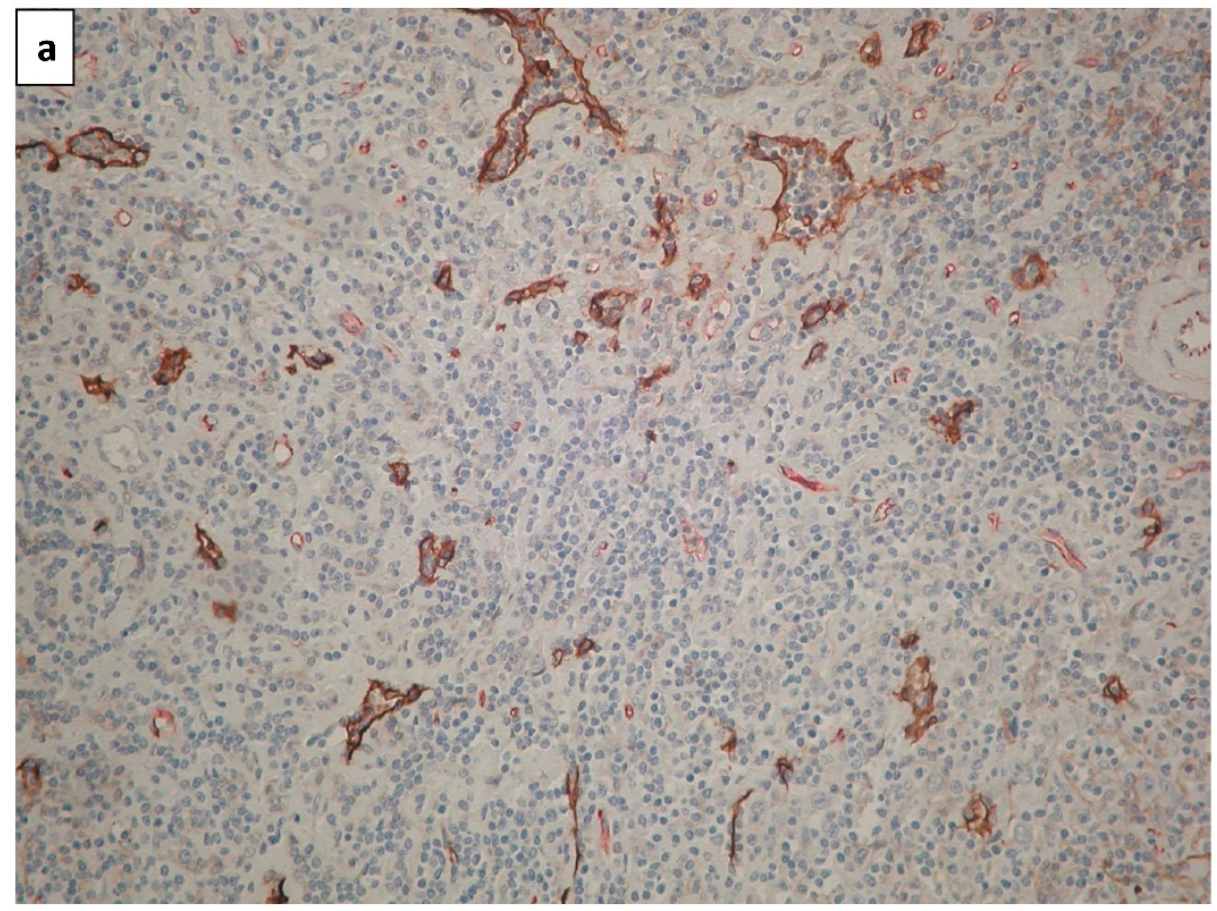



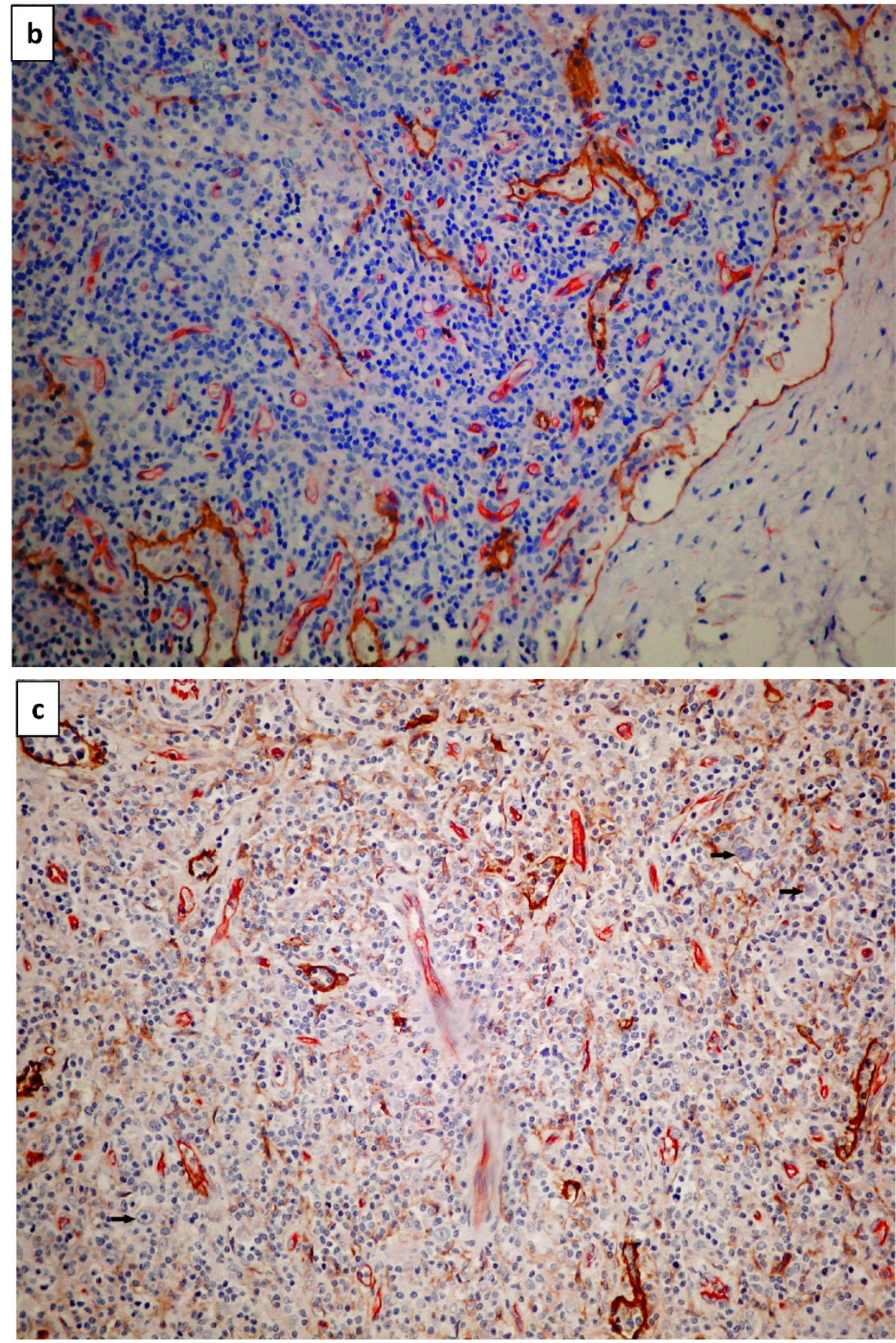

Figure 1. a. The photomicrograph of a classical Hodgkin lymphoma section, highlights predominant lymphatic vessels stained with anti-D2-40 (Podoplanin) in brown (IHC X340 with DAB substrate). b. Double staining of a section of classical lymphoma: blood vessels stain in pink-red with CD34 and lymphatic vessels in brown with D2-40. Note a residual subcapsular sinus on the right margin is highlighted in brown (IHCX340 with AEC for blood vessels and DAB for lymphatic vessels). c. Double staining of a section of classical lymphoma: blood vessels stain pink with CD34 and lymphatic vessels stain brown with D2-40. Note scarce Hodgkin (mononuclear) tumor cells (arrows) show no space association with lymphatic vessels (IHCX340).

No significant association was found between the four lymphatic parameters of cHL and the clinical features - age, gender, ethnicity, stage, outcome and death from the disease.

No correlation was evident with the following immune markers of cHL: CD30, EBV, CD20, p53, MDM-2 expression. Apoptotic index was not associated with the LV parameters. A significant negative relationship was found however between LV parameters and the pRB expression - with the mean lymphatic area and the mean complexity factor. BAX was inversely and significantly related with the mean surface area and the mean length. IкB- $\alpha$ was inversely and to a significant degree associated with the mean LV perimeter (Table 1 ). 
Table 1. Association between biological sample features and lymphatic vessels parameters.

\begin{tabular}{|c|c|c|c|c|c|}
\hline & & $\begin{array}{l}\text { Perimeter } \\
\text { Mean (SD) }\end{array}$ & $\begin{array}{l}\text { Area Mean } \\
\text { (SD) }\end{array}$ & $\begin{array}{l}\text { Length Mean } \\
\text { (SD) }\end{array}$ & $\begin{array}{l}\text { Complex } \\
\text { factor Mean } \\
\text { (SD) }\end{array}$ \\
\hline \multirow[t]{3}{*}{ p53 } & positive & 7.1 (3.7) & $2.2(2.5)$ & $2.2(0.9)$ & $13.9(3.0)$ \\
\hline & negative & $5.2(1.1)$ & $1.2(0.5)$ & $2.2(0.3)$ & $15.2(4.6)$ \\
\hline & $P$ value & $\mathrm{p}=0.5$ & $\mathrm{p}=0.5$ & $\mathrm{p}=0.7$ & $p=0.6$ \\
\hline \multirow[t]{3}{*}{ MDM-2 } & positive & $5.7(0.9)$ & $1.6(0.5)$ & $1,9(0.3)$ & 14.9 (3.7) \\
\hline & negative & $7.1(4.2)$ & $2.0(2.9)$ & $2.3(0.9)$ & $13.8(3.4)$ \\
\hline & $P$ value & $\mathrm{p}=0.9$ & $\mathrm{p}=0.1$ & $\mathrm{p}=0.6$ & $p=0.6$ \\
\hline \multirow[t]{2}{*}{ Apoptotic } & Index & $\mathrm{r}=-0.14$ & $\mathrm{r}=-0.12$ & $r=0.19$ & $\mathrm{r}=0.02$ \\
\hline & $\mathrm{P}$ value & $p=0.6$ & $p=0.7$ & $\mathrm{p}=0.5$ & $p=0.9$ \\
\hline \multirow[t]{3}{*}{$\mathrm{Rb}$} & positive & $5.9(3.5)$ & $1.1(0.5)$ & $1.9(0.5)$ & 14.5 \\
\hline & negative & 7.8 & 3.7 (3.7) & $2.8(1.0)$ & $11.4(0.7)$ \\
\hline & $\mathrm{P}$ value & $\mathrm{p}=0.1$ & $\mathrm{p}=0.02$ & $\mathrm{p}=0.08$ & $\mathrm{p}=0.034$ \\
\hline \multirow[t]{3}{*}{ BAX } & positive & $5.9(3.5)$ & $1.1(0.6)$ & $1.9(0.4)$ & $15.7(3.8)$ \\
\hline & negative & 7.1 & $2.9(3.1)$ & $2.6(0.9)$ & $12.3(1.7)$ \\
\hline & $\mathrm{P}$ value & $\mathrm{p}=0.9$ & $p=0.05$ & $\mathrm{p}=0.036$ & $p=0.066$ \\
\hline \multirow[t]{3}{*}{ ІкB- $\mathrm{a}$} & positive & $4.9(1.2)$ & $1.2(0.7)$ & $1.9(0.5)$ & $12.9(2.2)$ \\
\hline & negative & $8.2(3.8)$ & $2.5(2.9)$ & $2.4(0.9)$ & $15.9(4.2)$ \\
\hline & P value & $\mathrm{p}=0.014$ & $p=0.3$ & $\mathrm{p}=0.4$ & $\mathrm{p}=0.2$ \\
\hline
\end{tabular}

Of all the LV parameters, only the mean LV major axis length was inversely related with the shape factor, to a significant extent $(r=-.6 ; \mathrm{p}=.03)$.

The only significant association of the mean lymphatic perimeter was with the inverse ІкB- $\alpha$ expression in tumor cells $(\mathrm{p}=.015)$.

When relating the mean LV surface area with several cHL features, we only found an inverse significant relationship with $\mathrm{pRB}$ and BAX expression (Table 2).

Table 3 shows that the only significant association of the shape factor was with the pRB expression and it was direct. None of the lymphatic vessels showed in their lumina evidence of Hodgkin-Reed-Sternberg tumor cells of cHL.

\section{Relevance of expression of $p R b, B a x$ and IkB- $\alpha$}

We next analyzed the comprehensive relevance of the expression of $\mathrm{pRb}, \mathrm{BAX}$ and IKB- $\alpha$ in a larger cohort of classical HL patients.

The only significant link of $\mathrm{pRb}$ expression in the larger cohort of cHL patient was with FH6 expression, a sialylated-CD15 antigen, and it was negative $(p=$ .044). Clinically, BAX was inversely related with bulky HL $(p=.040)$. No other significant overall association was found with other clinical traits.

Table 4 shows associations between BAX expression and biological markers of cHL. It shows a positive relationship with MCL1, p53 and MDM2 expression, to a significant degree and a significant inverse link with BAK, and BCL2. Within this table is also a correlation of high significance with proteins of the measles virus. Using the Kaplan-Meier analysis, no significant difference was found in overall survival of positive and negative expression of BAX. The multivariable logistic regression relates the measles nucleoprotein and $\mathrm{BAX}-\mathrm{HR}=.643 ; \mathrm{p}=.004$.

Table 2. Association between lymphatic vessels area and Hodgkin lymphoma features.

\begin{tabular}{llllll}
\hline & & $\mathrm{n}$ & $\begin{array}{l}\text { Mean } \\
(\mathrm{SD})\end{array}$ & $\begin{array}{l}\text { Mean } \\
\text { Rank }\end{array}$ & p value \\
\hline Stage & I-IIA & 8 & $6.5(2.6)$ & 9.13 & \\
& IIB-IVB & 6 & $6.9(4.1)$ & 5.33 & 0.093 \\
$\mathrm{Rb}$ & Pos & 9 & $1.1(0.5)$ & 5.33 & \\
expression & Neg & 4 & $3.7(3.7)$ & 10.75 & 0.021 \\
Cas3 & Pos & 11 & $1.2(0.5)$ & 6.36 & \\
expression & Neg & 3 & $4.3(4.2)$ & 11.67 & 0.052 \\
BAX & Pos & 9 & $1.1(0.6)$ & 6.11 & \\
expression & Neg & 6 & $2.9(3.1)$ & 10.83 & 0.045 \\
IкB & Pos & 8 & $4.9(1.2)$ & 5.38 & .015 \\
express & neg & 7 & $8.2(3.4)$ & 11.00 & \\
\hline
\end{tabular}

Table 3. Association between the complexity factor of lymphatics and Hodgkin lymphoma features.

\begin{tabular}{llllll}
\hline & & $\mathrm{n}$ & Mean (SD) & Mean Rank p value \\
\hline B & Yes & 7 & $15.5(3.2)$ & 8.00 & \\
Symptoms & No & 5 & $12.6(1.7)$ & 4.40 & 0.088 \\
Bulky & Yes & 4 & $16.3(3.4)$ & 9.00 & \\
& No & 8 & $13.4(2.5)$ & 5.25 & 0.089 \\
Outcome & NED & 7 & $13.3(0.6)$ & 5.57 & \\
& AWD\&DOD & 7 & $15.6(3.2)$ & 9.43 & 0.097 \\
Relapse & Yes & 3 & $15.5(2.6)$ & 8.00 & \\
& No & 7 & $12.4(2.1)$ & 4.43 & 0.087 \\
Rb & Pos & 9 & $14.5(3.1)$ & 8.56 & \\
expression & Neg & 4 & $11.4(0.7)$ & 3.50 & 0.031 \\
Cas3 & Pos & 11 & $14.8(3.5)$ & 8.55 & \\
expression & Neg & 3 & $11.3(0.8)$ & 3.67 & 0.073 \\
BAX & Pos & 9 & $15.7(3.8)$ & 9.78 & \\
expression & Neg & 6 & $12.3(1.7)$ & 5.33 & 0.059 \\
\hline
\end{tabular}

NED - no evidence of disease. AWD \& DOD - alive with disease or dead of disease.

Table 4. BAX expression and biological characteristics of classical Hodgkin lymphoma.

\begin{tabular}{|c|c|c|c|}
\hline & BAX-pos n (\%) & BAX-neg n (\%) & p value \\
\hline \multicolumn{4}{|c|}{ BCL2, n=173 } \\
\hline Pos & $44(57.9)$ & 37 (38.1) & \\
\hline neg & $32(42.1)$ & $60(61.9)$ & .007 \\
\hline \multicolumn{4}{|c|}{ BAK, n=37 } \\
\hline Pos & $13(65)$ & $3(17.6)$ & \\
\hline neg & $7(35)$ & $14(82.4)$ & .005 \\
\hline \multicolumn{4}{|c|}{ MCL1, $n=30$} \\
\hline Pos & $13(76.5)$ & $5(38.5)$ & \\
\hline neg & $4(23.5)$ & $8(61.5)$ & .042 \\
\hline \multicolumn{4}{|c|}{ p53, n=178 } \\
\hline pos & $69(88.5)$ & $73(73)$ & \\
\hline neg & $9(11.5)$ & $27(27)$ & .008 \\
\hline \multicolumn{4}{|c|}{ MV,$n=128$} \\
\hline Pos & $51(81)$ & $49(64.5)$ & \\
\hline neg & $12(19)$ & $27(35.5)$ & .024 \\
\hline \multicolumn{4}{|c|}{ MV-NP, n=68 } \\
\hline Pos & $18(72)$ & $15(38.5)$ & \\
\hline neg & $7(28)$ & $24(61.5)$ & .030 \\
\hline \multicolumn{4}{|c|}{$\mathrm{pRb}, \mathrm{n}=102$} \\
\hline pos & $29(64.4)$ & $38(66.7)$ & \\
\hline neg & $16(35.6)$ & 19 (33.3) & .489 \\
\hline
\end{tabular}

MV - measles virus protein. MV-NP - Measles virus nucleoprotein. 
Table 5 reveals significantly inverse associations between IкB- $\alpha$ expression and the measles nucleoprotein and hemagglutinin. No variation was found between positive and negative expression of ІкB- $\alpha$, by Kaplan-Meier analysis. The multivariable logistic regression correlating with the measles nucleoprotein with IKB- $\alpha-\mathrm{HR}=.206 ; \mathrm{p}=.010$. In addition, an inverse relationship between this gene expression and the LMP1 of EBV is also significant. In addition, IкB- $\alpha$ was related significantly to two non-sialyl-CD15 antigens, LeuM1 and 80H5. No such relation was found with sialylated-CD15.

Table 5. IкB- $\alpha$ expression associated with biological features of classical Hodgkin lymphoma.

\begin{tabular}{llll}
\hline & IкBa-pos, n (\%) & IкBa-neg, $\mathrm{n}(\%)$ & $\mathrm{p}$ value \\
\hline LeuM1, n-174 & & & \\
Pos & $112(91.8)$ & $41(78.8)$ & \\
neg & $10(8.2)$ & $11(21.2)$ & .018 \\
80H5, n=174 & $110(90.2)$ & $41(78.8)$ & \\
Pos & $12(9.8)$ & $11(21.2)$ & .041 \\
neg & & & \\
LeX1, n=153 & $10(9.3)$ & $4(8.9)$ & \\
Pos & $98(90.7)$ & $41(91.1)$ & .605 \\
neg & & & \\
MV-Ha, n=137 & $32(31.7)$ & $5(13.9)$ & .029 \\
Pos & $69(68.3)$ & $31(86.1)$ & \\
neg & & \\
MV-NP, n=163 & $67(58.3)$ & $19(39.6)$ & .022 \\
Pos & $48(41.7)$ & $29(60.4)$ & \\
neg & & & \\
LMP1, n=176 & $46(37.1)$ & $9(17.3)$ & .007 \\
Pos & $78(62.9)$ & $43(82.7)$ & \\
neg & LeuM1 and 80H5 - antibodies against non-sialyl-CD15. LeX1 - against sialyl-CD15. \\
MV-Ha - Hemagglutinin measles protein. MV-NP - Measles virus nucleoprotein.
\end{tabular}

\section{Discussion}

Lymphangiogenesis has shown different properties and significance when compared with angiogenesis in primary epithelial malignancies, as well as in the related lymph node metastases [13, 14]. However, the theme of our paper is the nature of lymphangiogenesis in the malignant tumor, cHL, which develops primarily in lymph nodes. In addition, the mode of spread in $\mathrm{CHL}$, at least at the disease outset, is from one group of lymph nodes to its neighbor.

A single paper has approached the estimation of lymphangiogenesis in cHL, by assessing VEGF in cHL patients. These authors showed limited findings on the positive expression of VEGFC in Hodgkin-Reed-Sternberg cells, in the patients' sera as well as on its gene expression [9].

Since lymphangiogenesis in cancer seems to raise a debate on its possible role in various malignancies [15-17], we opted for studying its significance in $\mathrm{cHL}$ and also for the use the morphometric approach. Lymphatic vessels were defined by immunohistochemistry with the D2-40 (podoplanin) antibody and when relevant, with the double staining by including in addition the CD34 antibody.

In cHL, HRS cells may be numerous, but are far more often scarce $(0.1-1 \%)$ within a reactive cells background. We found no specific structural relationship between HRS cells and lymphatic vessels (Fig. 1c). As lymphangiogenesis was determined by identifying hot spots, no relation was found between them and the distribution of the HRS cells (which are dispersed in loose aggregates in the lymph node section). Of note, in the study of angiogenesis in cHL, no particular relation between blood vessels and HRS cells was described, to our knowledge.

Figure $1 \mathrm{~b}$, with its opened subcapsular sinus, is the nearest to a reactive lymph node, as the involvement with cHL is partial. The lymph vessels distribution is therefore variable.

We defined 4 morphometric parameters for each LV: its mean perimeter, its mean surface area, its mean major axis length and a calculated factor of complexity [8].

The number of cases studied was limited, due to the preliminary (pilot) nature of the study. But, each was studied twice and each case was represented by 3 different hot spots. We analyzed by morphometry 15 hot spots.

Our main positive findings concerned a statistically significant association between $\mathrm{pRb}$ expression and two LV parameters - the mean lymphatic area and the mean complexity factor, both inversely. Modulation of angiogenesis by $\mathrm{pRb}$ has been described in various tumors [18-20]. The involvement of the $\mathrm{pRb}$ pathway in angiogenesis have been demonstrated using the papillomavirus oncoproteins. However, this gene has not been associated with lymphangiogenesis.

BAX expression was negatively and significantly related with the mean LV surface area and the mean LV length. In contrast, VEGFC expression in carcinoma of the urinary bladder correlated directly with the BAX expression (21). It is of note that BAX expression is often correlated with that of bcl-2; bcl-X and Mcl-1 and is not necessarily correlated with the presence of apoptotic tumor cells [22], or with survival [23].

IKB- $\alpha$ was inversely and significantly associated with the mean LV perimeter. This gene has been considered as a regulator of angiogenesis, but no such relation has been recognized to date with lymphangiogenesis [24]. Thus inhibition of IkB-a may promote NF-KB signaling and together with it, angiogenesis. It remains to find out if 
lymphangiogenesis would also be activated by this association.

The only correlation found with the mean LV complexity factor was inverted and was of the mean LV major axis length. It is proposed that during a lymph vessel maturation, it may undergo modulation from a small and complex figure to a longer and simpler form.

However, most variables, clinical or biological were not associated with either of the LV morphometric characteristics.

On a wider perspective, anti-angiogenetic therapy may not have completely fulfilled all the expectations invested in it [4]. We have mentioned above the practical lack of documentation on lymphangiogenesis in cHL.

The impact of angiogenesis in cHL is also very limited, both in extent and in significance [7, 25-27]. Nevertheless, angiogenesis in cHL is not the issue here and no evidence is found that we can extrapolate these data to the status of lymphangiogenesis in this form of cancer. In any case no lymph vessel in our material contained cHL tumor cells.

In summary, the morphologic-pathologic pilot survey establishes in cHL a notable lymphangiogenesis which has not been demonstrated to date. The lymphatic vessels scrutinized herein showed variations in all their morphometric parameters. We found no clinical nor biological correlations with the lymphangiogenetic features displayed. The expression of three genes represented the major statistically significant findings of the study: a negative association between the LVs and $\mathrm{pRb}, \mathrm{BAX}$ and IкB- $\alpha$ and LV characteristics. Moreover, the relation between the three genes and $\mathrm{cHL}$, have been investigated in a second part of the paper, in a more extensive cohort of cHL patients [12].

The findings of this expanded study are segregated into three main topics, all integral parts of the $\mathrm{cHL}$ research. The most striking associations were with the expression of measles virus proteins, notably with BAX and IkBa expression, and therefore with lymphangiogenesis. We have noted extensively, in the past, an association between cHL and measles virus [28]. Moreover, a connection with apoptosis modulation as a possible mechanism, has been already raised [12]. We have also suggested that the major role, attributed to NF- $\mathrm{kB}$ in the pathogenesis of cHL, may not be absolute [28].

The second group of findings is related also with apoptosis regulation. They deal with BAX expression. Notably, the relation and associations of the members of the BCL2 family of proteins, are not straightforward, this finding has been established previously [22] and no relationship with cell survival is to be found [23]. It seems the overall level distribution of all the components of the BCL2 family is of more significance than the status of each individual member of the family. The activation of the different members may also be critical [29]. The association of the tumor cells apoptosis with lymphangiogenesis, although not immediate, is clearly present, if one recapitulates the results of the pilot study.

A third category of findings is related with the link between $\mathrm{pRb}$ and the sialyl-CD15, and that of IKB- $\alpha$ and the non-sialyl-CD15 antigens which carry an immunomorphologic relevance in this malignancy. This may represent a new association with the CD15 antigens, not described to date, which will need deciphering, in the context of lymphangiogenesis.

Thus, lymphangiogenesis in cHL may present various biological effects, which are not necessarily expected.

\section{Acknowledgements}

In Memoriam: Dr Isebrand Prinsloo was the initiator and motor of this study and he departed before he could finalize it.

We thank Kibbutz Sde-Boker; the Israel Science Foundation, the Israel Cancer Association, the Israel Ministry of Health and the Holzer Family Foundation for their partial support.

\section{Competing Interests}

The authors have declared that no competing interest exists.

\section{References}

1. Orecchioni S, Gregato G, Martin-Padura I, et al. Complementary populations of human adipose tissue CD34+ progenitor cells promote growth, angiogenesis, and metastasis of breast cancer. Cancer Res. 2013; 73: 5880-91.

2. Bing Z, Jian-ru Y, Yao-quan J, et al. Evaluation of angiogenesis in non-small cell lung carcinoma by CD34 immunohistochemistry. Cell Biochem Biophys. 2014; 70: 327-31.

3. Wong ML, Prawira A, Kaye AH, et al. Tumor angiogenesis: its mechanisms and therapeutic implications in malignant gliomas. Clin Neurosci. 2009; 16: 1119-30.

4. Jeong HS, Jones D, LJao S, et al. Investigation of the lack of angiogenesis in the formation of the lymph node metastasis. J Natl Cancer Inst. 2015; 107: pii:djv155.

5. Alitalo A, Detmar M. Interaction of tumor cells and lymphatic vessels in cancer progression. Oncogene. 2012; 31: 4499-508.

6. Oki Y, Copeland A, Younes A. Clinical development of panobinostat in classical Hodgkin lymphoma. Expert Rev Hematol. 2011; 4: 245-52.

7. Koh YW, Park C, Yoon DH, et al. Prognostic significance of Cox-2 expression and correlation with Bcl-2 and VEGF expression, microvessel density and clinical variables in classical Hodgkin lymphoma. Am J Surg Pathol. 2013; 37: 1242-51.

8. Korkolopoulou P, Thymara I, Kavantzas TP, et al. Angiogenesis in Hodgkin lymphoma: a morphometric approach in 286 patients with prognostic implications. Leukemia. 2005; 19: 894-90.

9. Rueda A, Olmos D, Vicioso L, et al. Role of vascular endothelial growth factor-C in classical Hodgkin lymphoma. Leuk Lymphoma. 2015; 56: 1286-94.

10. Benharroch D, Levy A, Prinsloo I, et al. Apoptotic index as a prognostic factor in Hodgkin's disease. Leuk Lymphoma. 1999; 33: 351-9.

11. Kyzas PA, Geleff S, Batistatou A, et al. Evidence of lymphangiogenesis and its prognostic implications in head and neck squamous cell carcinoma. J Pathol. 2005; 206: 170-7. 
12. Benharroch D, Einav I, Feldman A, et al. Apoptosis of Hodgkin-Reed-Sternberg cells in classical Hodgkin lymphoma revisited. APMIS. 2010; 118: 339-45.

13. Fujii $\mathrm{T}$, Sutoh $\mathrm{T}$, Morita $\mathrm{H}$, et al. Vascular invasion, but no lymphatic invasion, of the primary tumor is a strong prognostic factor in patients with colorectal carcinoma. Anticancer Res. 2014; 34: 3147-51.

14. Kanngurn S, Thongsukai $\mathrm{P}$, Chewatanakornkul S. Chalkey microvessel, but no lymphatic density correlates with axillary lymph node metastasis in primary breast cancers. Asian Pac J Cancer Prev. 2013; 14: 583-7.

15. Kostis G, Ioannis L, Helen K, et al. The expression of vascular endothelial growth factor- $\mathrm{C}$ correlates with lymphatic microvessel density and lymph node metastasis in prostate carcinoma. An immunohistochemical study. Urol Ann. 2014; 6: 224-30.

16. Zhao $\mathrm{YC}, \mathrm{Ni} \mathrm{XJ}, \mathrm{Li} \mathrm{Y}$, et al. Peritumoral lymphangiogenesis induced by vascular endothelial growth factor $C$ and $D$ promotes lymph node metastasis in breast cancer patients. World J Surg Oncol. 2012; 109: 165.

17. de Sousa SF, Gleber-Netto FO, de Oliveira-Netto $\mathrm{HH}$, et al. Lymphangiogenesis and podoplanin expression in oral squamous cell carcinoma and associated lymph nodes. Appl Immunohistochem Mol Morphol. 2012; 20: 588-94.

18. Dasgupta P, Sun J, Wang S, et al. Disruption of the Rb--Raf-1 interaction inhibits tumor growth and angiogenesis. Mol Cell Biol. 2004; 24: 9527-41.

19. Claudio PP, Stiegler $\mathrm{P}$, Howard $\mathrm{CM}$, et al. $\mathrm{Rb} 2 / \mathrm{p} 130$ gene enhanced expression down-regulates vascular endothelial growth factor expression and inhibits angiogenesis in vivo. Cancer Res. 2001; 61: 462-8.

20. Gaballini C, Del Bufalo D, Zupi G. Involvement of RB gene family in tumor angiogenesis. Oncogene. 2006; 25: 5326-32.

21. Mylona E, Magkou C, Gorantonakis G, et al. Evaluation of the vascular endothelial growth factor-C role in urothelial carcinomas of the bladder. Anticancer Res. 2006; 26: 3567-71.

22. Brousset $\mathrm{P}$, Benharroch $\mathrm{D}$, Krajewski $\mathrm{S}$, et al. Frequent expression of the cell death-inducing gene Bax in Reed-Sternberg cells of Hodgkin's disease. Blood. 1996; 87: 2470-5.

23. Rassidakis GZ, Medeiros LJ, McDonnell TJ, et al. Bax expression in Hodgkin and Reed-Sternberg cells of Hodgkin's disease: correlation with clinical outcome. Clin Cancer Res. 2002; 8: 488-93.

24. Coles AH, Gannon H, Cerny A, et al. Inhibitor of growth-4 promotes IkappaB promotor activation to suppress NF-kappaB signaling and innnate immunity. Proc Natl Acad Sci USA. 2010; 107: 11423-8.

25. Koh YW, Park CS, Yoon DH, et al. CD163 expression was associated with angiogenesis and shortened survival in patients with uniformly treated classical Hodgkin lymphoma. PLoS One. 2014; 9: e87066.

26. Panico L, Ronconi F, Lepore M, et al. Prognostic role of tumor associated macrophages and angiogenesis in classical Hodgkin lymphoma. Leuk Lymphoma. 2013; 54: 2418-25.

27. Dimtsas GS, Georgiadi EC, Karakisos P, et al. Prognostic significance of IHC expression of the angiogenic molecules vascular endothelial growth factor-A, vascular endothelial growth factor receptor-2 in patients with classical Hodgkin lymphoma. Leuk Lymphoma. 2014; 55: 558-64.

28. Benharroch D, Gopas J, Ariad S. Does the measles virus contribute to carcinogenesis? A review. J Cancer. 2014; 5: 98-102.

29. Iyer S, Anwari K, Alsop AE, et al. Identification of an activation site in BAK and mitochondrial BAX triggered by antibodies. Nat Commun. 2016; 7: 11734 . 